\title{
K. Alex Müller: in Honor of His 90th Birthday
}

\author{
Annette Bussmann-Holder ${ }^{1} \cdot$ Hugo Keller $^{2}$
}

Received: 25 May 2017 / Accepted: 19 June 2017 / Published online: 13 July 2017

(c) Springer Science+Business Media, LLC 2017

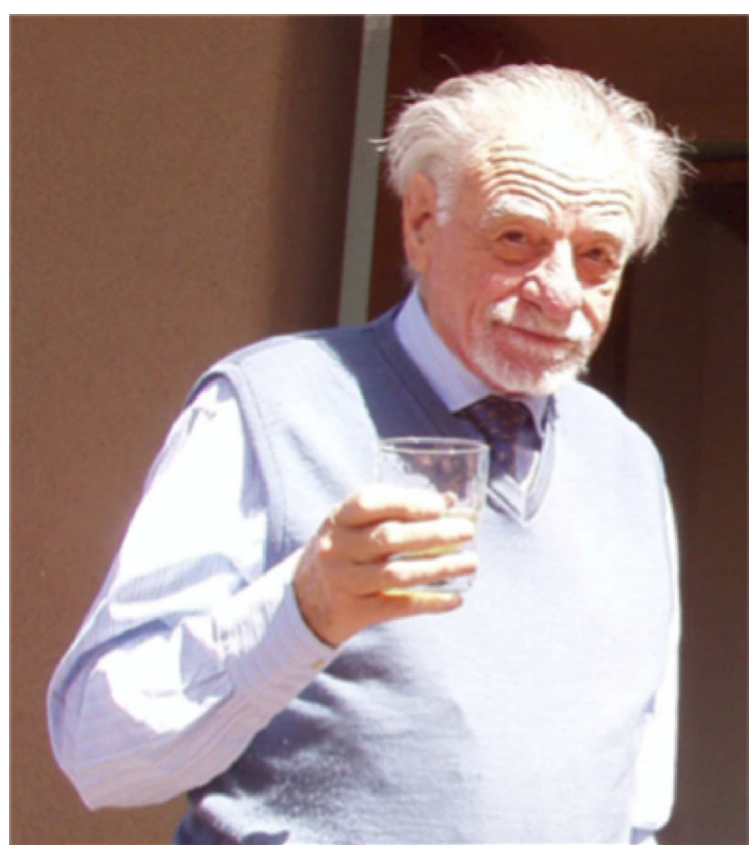

It is our great pleasure to write a short review on the scientific career of the jubilee. The readership of this journal, where K. A. Müller is honorary editor, might not be familiar with the fact that long before the breaking-through discovery

Annette Bussmann-Holder

a.bussmann-holder@fkf.mpg.de

1 Max-Planck-Institute of Solid State Research, Stuttgart, Germany

2 Physik-Institut der Universität Zürich, Zürich, Switzerland of high-temperature superconductivity by J. Georg Bednorz and K. Alex Müller, which was awarded by the Nobel Prize only one year after its discovery, Alex was already famous in the scientific world. Several times he mentioned to us that he could have finished his activities just before the discovery and start a life as administrative director at IBM, since he had achieved already almost everything on his personal to-do list. As is well known, he did not follow this perspective, but stayed with science. His scientific activities which finally led to the Nobel Prize were deeply ingrained in the research of perovskite oxides. He received worldwide acknowledgement with his outstanding exploration of the phase transitions in these compounds by using the electron paramagnetic resonance (EPR) technique. His data and their interpretation provided the key information about the microscopic mechanism of the structural phase transitions where the oxygen octahedral rotational instability was shown to be the driving force behind it. They also evidenced unambiguously that in $\mathrm{SrTiO}_{3}$ indeed a structural phase transition from cubic to tetragonal takes place at $105 \mathrm{~K}$, which in view of the tiny distortion was extremely difficult to observe. Besides this important advancement, the low-temperature properties of $\mathrm{SrTiO}_{3}$ were in his focus where he discovered a novel state of matter, namely quantum paraelectricity. This concept is based on the fact that strong transverse optic mode softening takes place in this compound, reminiscent of a ferroelectric instability. However, quantum fluctuations inhibit the polar instability and induce a saturation of the mode frequency. This phenomenon is rather rare, but has been observed also in later on discovered socalled incipient ferroelectrics. While Alex's experimental research on $\mathrm{SrTiO}_{3}$ has induced worldwide activities and has important consequences not only from a scientific point of view, but also for applications, also his theoretical work 
on phase transitions and the Jahn-Teller effect provided substantial impact to the understanding of critical phenomena, electron-lattice interactions, and competing and coexisting order parameters. Importantly he suggested that rotational fluctuations well above a structural instability give rise to a crossover from fast to slow fluctuations upon approaching the transition. An essential concept was introduced by him in more recent years when he reinvestigated the scheme of order/disorder versus displacive transitions. Interestingly, he established that there is no sharp transition line between both, but that a coexistence of them is possible where different time and length scales become relevant. This important issue also clarifies that different experimental techniques might arrive at seemingly controversial results, since the probing time and length scales are discriminative. It emphasizes in addition that experiments can be complementary and thus enrich the viewpoints on a specific material.

Besides the classical perovskite-based ferroelectrics, Alex also worked in the field of hydrogen-bonded ferroelectrics which have long been believed to order due to the hydrogen bond ordering. For this material class, he demonstrated that locally polarized clusters form already far above the critical phase transition temperature and exhibit distinct life times. Since these clusters develop independently from the proton tunneling motion, it was the first evidence that the phase transition mechanism in these complex materials is strongly driven by the heavy ion sublattice.

Superconductivity was for a long time a field which he avoided, mainly because-according to his own wordshe did not understand it. He became interested in it during his stay at IBM in Yorktown Heights where he recognized that granular $\mathrm{Al}$ has a much higher superconducting transition temperature than crystalline $\mathrm{Al}$ and that it is strongly inhomogeneous with locally varying transition temperatures and superconducting gaps. The locking of phases between the grains is the decisive process to reach at lower temperatures a homogenous superconducting phase. While the concept of inhomogeneity in the context of superconductivity was novel at that time, it is now an important ingredient in understanding high-temperature superconductivity.

The way to the discovery of high-temperature superconductivity was rather cumbersome and accompanied by various disappointments, even though the route was well designed. The basic idea of Alex was to look for superconductivity in oxides, materials which have been discarded in the search for it. He made the important observation that the few superconducting oxides, known at that time, had an irritating small density of states at the Fermi energy. In order to explain their rather large transition temperatures, a very strong electron-phonon interaction had to be presumed. This, in turn, easily leads to localization and thus a breakdown of superconductivity. Thus, an additional ingredient was needed which he found in the work by Harry Thomas and coworkers, namely the dynamic JahnTeller polaron. The fact that its energy is not bound to typical lattice vibrations provided a pathway to new coupling schemes extending the conventional pairing glue. In spite of this clear concept, many attempts were needed to finally arrive at the cuprates. It is noteworthy to mention that both K. Alex Müller and J. Georg Bednorz did not expect to really discover high-temperature superconductivity exceeding liquid nitrogen temperature, but to show that oxides with Jahn-Teller active ions are candidates for elevated superconducting transition temperatures.

After the breakthrough in 1986, the life of Alex changed dramatically and was dominated by many honors with the upmost honor of the Nobel Prize in 1987. Nevertheless, he continued former research projects and initiated novel ones, where the probably most important one was the research of isotope effects in copper oxides which was carried out at the University of Zürich. Isotope effects have been observed not only on the superconducting transition temperature but also on the penetration depth, the superconducting energy gaps, the Néel temperature of the antiferromagnetic parent compounds, the so-called pseudo-gap formation temperature, and throughout the whole phase diagram. These partly very unconventional effects could all be consistently explained within the original idea behind the discovery, namely polaron and bipolaron formation. Another aspect which he pursued was the notion of inhomogeneity and its role in copper oxides. While the overall opinion was that these materials are pure d-wave superconductors, Alex recognized soon that many experiments are inconsistent with this assumption and showed that a substantial s-wave component has to be present as well, which then leads in a natural way to inhomogeneity. In spite of the majority belief that high-temperature superconductivity in copper oxides is due to a magnetic pairing glue in a homogenous material, Alex convincingly demonstrated that his original idea of Jahn-Teller polaron formation leading to the discovery is in convincing agreement with most experimental facts.

His research activities and interests remained ongoing, and even at the age of 90, he still follows new progresses and discoveries. His proposals and suggestions for new projects are promising and surely need to be taken up.

As must be obvious from the above very short and incomplete description of Alex's scientific achievements, he substantially contributed to solid state physics in various areas with groundbreaking new discoveries and ideas. Besides these merits, it is important to emphasize that he 
was always interested in supporting young people, $\mathrm{PhD}$ students, and postdocs. He actively encouraged them to pursue their own ideas and supported their careers. Attending as many seminar talks as possible at the university, he was well familiar with their individual research fields and always open for discussing these with them. In addition, he happily joined personal celebrations and regular parties at the Physik-Institut, thus being well aware of the personal lifestyle of the institute members.

We both also experienced his friendship and deep interest in our research and our life and families. Many times his advices helped substantially in overcoming a crisis, and discussing physics with him was and is a constant source of inspiration. 